\title{
Relaxed Cutting Plane Method for Solving Linear Semi-Infinite Programming Problems ${ }^{1}$
}

\author{
S. Y. WU, ${ }^{2}$ S. C. FANG, ${ }^{3}$ AND C. J. LIN ${ }^{4}$ \\ Communicated by $\mathbf{P}$. Tseng
}

\begin{abstract}
One of the major computational tasks of using the traditional cutting plane approach to solve linear semi-infinite programming problems lies in finding a global optimizer of a nonlinear and nonconvex program. This paper generalizes the Gustafson and Kortanek scheme to relax this requirement. In each iteration, the proposed method chooses a point at which the infinite constraints are violated to a degree, rather than a point at which the violations are maximized. A convergence proof of the proposed scheme is provided. Some computational results are included. An explicit algorithm which allows the unnecessary constraints to be dropped in each iteration is also introduced to reduce the size of computed programs.
\end{abstract}

Key Words. Linear semi-infinite programming, cutting plane method.

\section{Introduction}

Consider the following linear semi-infinite programming problem:

$$
\begin{aligned}
\text { (LSIP) } \min & \sum_{j=1}^{n} c_{j} x_{j}, \\
\text { s.t. } & \sum_{j=1}^{n} x_{j} f_{j}(t) \geq g(t), \quad \forall t \in T, \\
& x_{j} \geq 0, \quad j=1, \ldots, n,
\end{aligned}
$$

'This research was partially supported by the North Carolina Supercomputing Center, Cray Research Grant, and a National Textile Center Research Grant.

${ }^{2}$ Professor, Department of Mathematics, National Cheng Kung University, Tainan, Taiwan. ${ }^{3}$ Walter Clark Professor, Industrial Engineering and Operations Research, North Carolina State University, Raleigh, North Carolina.

${ }^{4}$ Graduate Student, Industrial and Operations Engineering, University of Michigan, Ann Arbor, Michigan. 
where $T$ is a compact metric space with an infinite cardinality, $f_{j}, j=1, \ldots, n$, and $g$ are real-valued continuous functions defined on $T$. Note that $T$ can be extended to a compact Hausdorff space (Refs. 1 and 2) without much difficulty.

Its dual problem can be formulated in the following form:

$$
\begin{aligned}
\text { (DLSIP) } \max & \int_{T} g(t) d \mu(t), \\
\text { s.t. } & \int_{T} f_{j}(t) d \mu(t) \leq c_{j}, \quad j=1, \ldots, n, \\
& \mu \in M^{+}(T),
\end{aligned}
$$

where $M^{+}(T)$ is the space of all nonnegtive bounded regular Borel measures on $T$.

Under some regularity conditions, it can be shown (Refs. 1 and 2) that there is no duality gap between (LSIP) and (DLSIP) and the latter achieves its optimum at an extreme point of its feasible domain. The applications of the linear semi-infinite programming can be referred to Refs. 3 and 4.

Many papers (Refs. 1, 3, 5,6) have dealt with solution methods for solving (LSIP). According to a recent review article (Ref. 7), the so-called cutting plane method or implicit exchange method is one of the key solution techniques. Basically, this approach finds a sequence of optimal solutions of corresponding regular linear programs in a systematic way and shows that the sequence converges to an optimal solution of (LSIP).

More precisely, in the $k$ th iteration, let

$$
T_{k}=\left\{t_{1}, t_{2}, \ldots, t_{k}\right\} \subset T,
$$

and consider the following linear program:

$$
\begin{aligned}
\left(\mathrm{LP}_{k}\right) \min & \sum_{j=1}^{n} c_{j} x_{j}, \\
\text { s.t. } & \sum_{j=1}^{n} x_{j} f_{j}\left(t_{i}\right) \geq g\left(t_{i}\right), \quad i=1,2, \ldots, k, \\
& x_{j} \geq 0, \quad j=1,2, \ldots, n .
\end{aligned}
$$

Firstly, we solve $\left(\mathrm{LP}_{k}\right)$ for an optimal solution

$$
\mathrm{x}^{k}=\left(x_{1}^{k}, x_{2}^{k}, \ldots, x_{n}^{k}\right)^{T} \text {. }
$$

Then, define

$$
\phi_{k}(t)=\sum_{j=1}^{n} f_{j}(t) x_{j}^{k}-g(t)
$$


Secondly, we find an optimizer

$$
t_{k+1} \in \arg \min _{t \in T} \phi_{k}(t)
$$

If

$$
\phi_{k}\left(t_{k+1}\right)=\sum_{j=1}^{n} f_{j}\left(t_{k+1}\right) x_{j}^{k}-g\left(t_{k+1}\right) \geq 0,
$$

then $x^{k}$ must be an optimal solution to (LSIP) because $\left(\mathrm{LP}_{k}\right)$ 's feasible domain contains (LSIP)'s feasible domain. Otherwise, we let

$$
T_{k+1}=T_{k} \cup\left\{t_{k+1}\right\}
$$

to construct $\left(\mathrm{LP}_{k+1}\right)$ and continue the iterative process. The convergence proof of $\left\{\mathbf{x}^{1}, \mathbf{x}^{2}, \ldots, \mathbf{x}^{k}, \ldots\right\}$ to an optimal solution of (LSIP) can be found in Ref. 3. A refined version which allows us to drop some redundant points in $T_{k}$ in order to reduce the size of $\left(\mathrm{LP}_{k}\right)$ can be found in Ref. 2 .

In the above approach, one constraint (corresponding to one cut) is added at a time and the major computational work in each iteration involves (i) solving a linear program $\left(\mathrm{LP}_{k}\right)$ and (ii) finding a global minimizer $t_{k+1}$ of $\phi_{k}(t)$. To reduce the computational requirement for solving $\left(\mathrm{LP}_{k}\right)$, an inexact approach was proposed earlier (Refs. 8 and 9). However, when the dimensionality of the compact metric space $T$ becomes high, finding a minimizer of a continuous function $\phi_{k}(t)$ over $T$ could be extremely timeconsuming, in particular, when $f_{j}(t)$ and $g(t)$ are highly nonlinear and nonconvex. In this case, the computational bottleneck of the cutting plane approach falls in finding a global minimizer $t_{k+1}$ of $\phi_{k}(t)$.

Ideas of relaxing the requirement of finding the global minimizer for different settings can be found in Refs. 10-13. For these approaches, at each iteration, instead of finding the global minimizer $t_{k+1}$ of $\phi_{k}(t)$, one either has to check that

$$
\phi_{k}(t) \geq 0, \quad \forall t \in T,
$$

in order to add a constraint (Ref. 10), or has to find the value

$$
\delta\left(x^{k}\right)=\min _{t \in T}\left\{\phi_{k}(t)\right\}
$$

in order to find a new cut at $t_{k+1}$ such that

$$
\phi_{k}\left(t_{k+1}\right)<0, \quad \phi_{k}\left(t_{k+1}\right) \leq \delta\left(x^{k}\right)+\epsilon_{k},
$$

where $\left\{\epsilon_{k}\right\}$ is a given sequence of nonnegative numbers converging to zero as $k$ increases to infinity (Refs. 12 and 13). Even so, the required computation could still be a bottleneck. 
In this paper, we further relax the Gustafson and Kortanek scheme such that a new cut is found at any $t_{k+1} \in T$ with $\phi_{k}\left(t_{k+1}\right)<-\delta$, where $\delta>0$ is a sufficiently small number which can be prescribed. In this way, we can avoid the task of finding the global minimizer $t_{k+1}$ and/or checking the minimum value $\delta\left(x^{k}\right)$ in every iteration. After introducing the relaxed scheme in Section 2, we show that, under appropriate conditions, the proposed scheme terminates in finite iterations to generate an approximate solution with desired accuracy. Some computational experiments and analysis are included in Section 3. Based on the relaxed scheme, an explicit algorithm which allows the unnecessary constraints to be dropped in each iteration is developed in Section 4, while some concluding remarks are made in Section 5.

\section{Relaxed Approach}

Given that $\delta>0$ is a prescribed small number, a general scheme is proposed as follows.

Step 1. Set $k \leftarrow 1$, choose any $t_{1} \in T$, and set $T_{1}=\left\{t_{1}\right\}$.

Step 2. Solve $\left(\mathrm{LP}_{k}\right)$ with an optimal solution $\mathrm{x}^{k}=\left(x_{1}^{k}, \ldots, x_{n}^{k}\right)^{T}$. Define $\phi_{k}(t)$ according to (7).

Step 3. Find any $t_{k+1} \in T$ such that $\phi_{k}\left(t_{k+1}\right)<-\delta$.

If such $t_{k+1}$ does not exist, stop and output $\mathrm{x}^{k}$ as the solution. Otherwise, set $T_{k+1}=T_{k} \cup\left\{t_{k+1}\right\}$.

Step 4. Update $k \leftarrow k+1$, and go to Step 2 .

Note that, if $\left(\mathrm{LP}_{k}\right)$ is found to be infeasible in Step 2, then (LSIP) is infeasible. In this case, there is no reason to continue the iterations. Therefore, without loss of generality, we may assume that $\left(L_{k}\right)$ is feasible in this paper. Also note that $t_{k+1} \notin T_{k}$ and, if the scheme terminates in Step 3, then the output solution $\mathrm{x}^{k}$ indeed solves (LSIP) with $\delta$ being small enough (up to machine accuracy, say $10^{-7}$ ). More on the effects of the size of $\delta$ will be discussed later. Moreover, the linear dual of $\left(\mathbf{L P}_{k}\right)$ can be formulated as the following problem:

$$
\begin{array}{rlr}
\left(\mathrm{DLP}_{k}\right) \max & \sum_{i=1}^{k} g\left(t_{i}\right) y_{i}, & \\
\text { s.t. } & \sum_{i=1}^{k} f_{j}\left(t_{i}\right) y_{i} \leq c_{j}, & j=1,2, \ldots, n, \\
& y_{i} \geq 0, & i=1,2, \ldots, k .
\end{array}
$$


For the purpose of easy description of the proposed approach, we assume that $\left(\mathrm{LP}_{k}\right)$ is solvable with an optimal value denoted by $V\left(\mathrm{LP}_{k}\right)$ and that $\left(\mathrm{DLP}_{k}\right)$ is also solvable with an optimal value denoted by $V\left(\mathrm{DLP}_{k}\right)$. Recall that $\mathrm{x}^{k}=\left(x_{1}^{k}, \ldots, x_{n}^{k}\right)^{T}$ solves $\left(\mathrm{LP}_{k}\right)$. Let

$$
B_{k}=\left\{j_{1}^{k}, \ldots, j_{p_{k}}^{k}\right\}
$$

be an index set such that

$$
x_{j}^{k}>0, \quad \text { if and only if } \quad j \in B_{k} .
$$

Then, we have the following theorem.

Theorem 2.1. If $\left(\mathrm{DLP}_{k+1}\right)$ is nondegenerate, then $V\left(\mathrm{LP}_{k+1}\right)>$ $V\left(\mathrm{LP}_{k}\right)$.

Proof. Define a $k \times n$ matrix $A$ with $a_{i j}=f_{j}\left(t_{i}\right)$ being its $(i, j)$ th element, for $i=1, \ldots, k$ and $j=1, \ldots, n$. Denoting

$$
\mathrm{b}=\left(g\left(t_{1}\right), \ldots, g\left(t_{k}\right)\right)^{T},
$$

we can rewrite $\left(\mathrm{LP}_{k}\right)$ in the following form:

$$
\begin{aligned}
\left(\mathrm{LP}_{k}\right) \min & \sum_{j=1}^{n} c_{j} x_{j}, \\
\text { s.t. } & {\left[A \mid-I_{k \times k}\right]\left[\begin{array}{c}
\mathrm{x} \\
\mathrm{x}^{\prime}
\end{array}\right]=\mathrm{b}, }
\end{aligned}
$$

$x, x^{\prime} \geq 0$.

Let

$$
\overline{\mathrm{x}}^{k}=\left[\begin{array}{c}
\mathrm{x}^{k} \\
\mathrm{x}^{* k}
\end{array}\right]
$$

be an optimal solution with a basis $B$. Its corresponding optimal basic variables are

$$
\overline{\mathrm{x}}_{B}^{k}=B^{-1} \mathrm{~b} \geq 0 .
$$

From the choice of $t_{k+1}$ in Step 3 of the proposed scheme, we have

$$
\begin{aligned}
\phi_{k}\left(t_{k+1}\right) & =\sum_{j=1}^{n} f_{j}\left(t_{k+1}\right) x_{j}^{k}-g\left(t_{k+1}\right) \\
& =\sum_{j \in B_{k}} f_{j}\left(t_{k+1}\right) x_{j}^{k}-g\left(t_{k+1}\right)<-\delta .
\end{aligned}
$$


If we let

$$
f=\left(f_{1}\left(t_{k+1}\right), \ldots, f_{n}\left(t_{k+1}\right)\right),
$$

then $\left(\mathrm{LP}_{k+1}\right)$ becomes

$$
\begin{aligned}
& \left(\mathrm{LP}_{k+1}\right) \min \sum_{j=1}^{n} c_{j} x_{j} \\
& \text { s.t. }\left[\begin{array}{c:c}
A & -I_{(k+1) \times(k+1)}
\end{array}\right]\left[\begin{array}{l}
\mathrm{x} \\
\mathrm{x}^{\prime \prime}
\end{array}\right]=\left[\begin{array}{l}
\mathrm{b} \\
\hdashline g\left(t_{k+1}\right)
\end{array}\right] \text {, } \\
& x, x^{\prime \prime} \geq 0 \text {. }
\end{aligned}
$$

Denoting

$$
\mathrm{c}=\left(c_{1}, \ldots, c_{n}\right)^{T},
$$

we have a linear dual program,

$$
\begin{aligned}
& \left(\mathrm{DLP}_{k+1}\right) \max \sum_{i=1}^{k+1} g\left(t_{i}\right) y_{i} \\
& \text { s.t. }\left[\begin{array}{c:c}
A & \\
\hdashline f & -I_{(k+1) \times(k+1)}
\end{array}\right]^{T} \mathrm{y} \leq\left[\begin{array}{l}
\mathrm{c} \\
\hdashline 0_{(k+1) \times 1}
\end{array}\right] \text {. }
\end{aligned}
$$

Moreover, we let

$$
f^{\prime}=\left(f_{1}\left(t_{k+1}\right), \ldots, f_{n}\left(t_{k+1}\right), 0, \ldots, 0\right) \in R^{n+k},
$$

and let

$$
f_{B}^{\prime}=\left(f_{j_{1}^{k}}^{k}\left(t_{k+1}\right), \ldots, f_{j_{p_{k}}^{k}}\left(t_{k+1}\right), 0, \ldots, 0\right)
$$

be those in $f^{\prime}$ corresponding to $B$. In this way, if

$$
\left[\begin{array}{c:c}
B & 0_{k \times 1} \\
\hdashline f_{B}^{\prime} & -1
\end{array}\right]\left[\begin{array}{l}
\overline{\mathrm{x}}_{B}^{k} \\
\hdashline \mathrm{x}^{*}
\end{array}\right]=\left[\begin{array}{l}
\mathrm{b} \\
\hdashline g\left(t_{k+1}\right)
\end{array}\right],
$$

then by (12),

$$
\mathrm{x}^{*}=\sum_{j \in B_{k}} f_{j}\left(t_{k+1}\right) x_{j}^{k}-g\left(t_{k+1}\right)=\phi_{k}\left(t_{k+1}\right)<-\delta .
$$

Moreover,

$$
\left[\begin{array}{c}
\overline{\mathrm{x}}_{B}^{k} \\
\hdashline \mathrm{x}^{*}
\end{array}\right]=\left[\begin{array}{c:c}
B & 0_{k \times 1} \\
\hdashline f_{B}^{\prime} & -1
\end{array}\right]^{-1}\left[\begin{array}{l}
\mathrm{b} \\
\hdashline g\left(t_{k+1}\right)
\end{array}\right] .
$$


Let

$$
\mathrm{y}^{*}=\left(y_{1}^{*}, \ldots, y_{k}^{*}\right)^{T}=\left(B^{-1}\right)^{T} c_{B} ;
$$

the theory of linear programming (Refs. 14 and 15) implies that $y^{*}$ is an optimal solution of $\left(\mathbf{D L} \mathbf{P}_{k}\right)$. Note that

$$
\left[\left[\begin{array}{c:c}
B & 0_{k \times 1} \\
\hdashline f_{B}^{\prime} & -1
\end{array}\right]^{-1}\right]^{T}\left[\begin{array}{c}
c_{B} \\
0
\end{array}\right]=\left[\begin{array}{c}
\mathrm{y}^{*} \\
0
\end{array}\right] .
$$

Hence, we know that

$$
\left[\begin{array}{c:c}
A & \\
\hdashline f & -I_{(k+1) \times(k+1)}
\end{array}\right]^{T}\left[\begin{array}{c}
\mathrm{y}^{*} \\
0
\end{array}\right] \leq\left[\begin{array}{l}
\mathrm{c} \\
\hdashline 0(k+1) \times 1
\end{array}\right] .
$$

In other words, $\left[\begin{array}{c}\mathrm{y}^{*} \\ 0\end{array}\right]$ is a feasible solution of $\left(\mathrm{DLP}_{k+1}\right)$ with a corresponding basic solution $\left[\begin{array}{c}x_{B}^{k} \\ \hdashline x^{*}\end{array}\right]$ for $\left(\operatorname{LP}_{k+1}\right)$.

Now, since

$$
\sum_{i=1}^{k} g\left(t_{i}\right) y_{i}^{*}+g\left(t_{k+1}\right) \cdot 0=V\left(\mathrm{DLP}_{k}\right)
$$

$\left(\mathrm{DLP}_{k+1}\right)$ is nondegenerate, and since

$$
\mathrm{x}^{*}<-\delta<0 \text {, }
$$

the basic property of the dual simplex method (Ref. 15, p. 97) guarantees that

$$
V\left(\mathrm{LP}_{k+1}\right)=V\left(\mathrm{DLP}_{k+1}\right)>V\left(\mathrm{DLP}_{k}\right)=V\left(\mathrm{LP}_{k}\right)
$$

The incremental amount in Theorem 2.1 can be further analyzed. Let

$$
\mathrm{y}^{k}=\left(y_{1}^{k}, \ldots, y_{k}^{k}\right)^{r}
$$

be an optimal solution of $\left(D L P_{k}\right)$. We define a discrete measure $\mu_{k}$ on $T$ such that

$$
\mu_{k}(t)= \begin{cases}y_{i}^{k}, & \text { if } t=t_{i} \in T_{k}, \\ 0, & \text { if } t \in T \backslash T_{k}\end{cases}
$$

In this way,

$$
\mu_{k}(t) \geq 0, \quad \forall t \in T .
$$


Furthermore, let

$$
T_{k}^{\prime}=\left\{t \in T_{k} \mid \mu_{k}(t)>0\right\},
$$

and let

$$
B_{k}^{\prime}=\left\{i_{1}^{k}, \ldots, i_{m_{k}}^{k}\right\}
$$

be an index set such that

$$
\mu_{k}\left(t_{i}\right)>0, \quad \text { if and only if } \quad i \in B_{k}^{\prime} .
$$

In other words,

$$
y_{i}^{k}>0, \quad \text { if and only if } \quad i \in B_{k}^{\prime} .
$$

We claim that

$$
t_{k+1} \in T_{k+1}^{\prime} .
$$

Note that, if $t_{k+1} \notin T_{k+1}^{\prime}$, then $\mu_{k+1}\left(t_{k+1}\right)=0$. In this case, the measure $\mu_{k+1}$ achieves nonzero value only at those points in $T_{k}$. Hence, we have

$$
V\left(\mathrm{DLP}_{k+1}\right)=V\left(\mathrm{DLP}_{k}\right),
$$

which contradicts Theorem 2.1. Therefore, without loss of generality, we can rearrange $t_{k+1}$ to be the last element in $T_{k+1}^{\prime}$. We define an $n \times m_{k}$ matrix $H_{k}$ with its $j$ th row vector being

$$
\left(f_{j}\left(t_{i \uparrow}^{k}\right), \ldots, f_{j}\left(t_{i_{m_{k}}^{k}}\right)\right), \quad j=1, \ldots, n .
$$

Since $\mu_{k}\left(t_{i}\right)>0, \forall i \in B_{k}^{\prime}$, from the complementary slackness theorem of linear programming (Refs. 14 and 15 ), we have

$$
H_{k}^{T} \mathrm{x}^{k}=\mathrm{g}^{k},
$$

where

$$
\mathrm{g}^{k}=\left(g\left(t_{i}^{k}\right), \ldots, g\left(t_{i_{m_{k}}^{k}}\right)\right)^{T} .
$$

Let $M_{k}$ be a $p_{k} \times m_{k}$ matrix with its $j$ th row vector being

$$
\left(f_{j}\left(t_{i \uparrow}^{k}\right), \ldots, f_{j}\left(t_{i_{m_{k}}^{k}}\right)\right), \quad j \in B_{k} .
$$

Recall that $t_{k+1}$ is the last element in $T_{k+1}^{\prime}$. Now, define an $n \times\left(m_{k}+1\right)$ matrix $\mathrm{H}_{k^{+}}$with its $j$ th row vector being

$$
\left(f_{j}\left(t_{i^{k}}\right), \ldots, f_{j}\left(t_{i_{m_{k}}^{k}}\right), f_{j}\left(t_{k+1}\right)\right), \quad j=1, \ldots, n .
$$

For

$$
\mathrm{g}^{k+1}=H_{k^{+}}^{T} \mathrm{x}^{k+1}
$$


from the constraints satisfied, we know that

$$
\left(\mathrm{g}^{k+1}\right)^{T} \geq\left(\left(\mathrm{g}^{k}\right)^{T}, g\left(t_{k+1}\right)\right) \equiv\left(\overline{\mathrm{g}}^{k+1}\right)^{T} .
$$

We define

$$
\mathrm{s}^{k}=\mathrm{g}^{k+1}-\overline{\mathrm{g}}^{k+1}
$$

Then,

$$
s^{k} \geq 0
$$

Moreover, the last element of $s^{k}$ must be zero. Otherwise, we have $\mu_{k+1}\left(t_{k+1}\right)=0$, which contradicts the fact that $\mu_{k+1}\left(t_{k+1}\right)>0$. Recalling the definition of $\phi_{k}(t)$, on the dual side we define

$$
\phi_{j}^{k}=\sum_{i \in B_{k}^{*}} f_{j}\left(t_{i}\right) \mu_{k}\left(t_{i}\right)-c_{j}, \quad j=1, \ldots, n .
$$

Note that

$$
\phi_{j}^{k} \leq 0, \quad \forall j, k,
$$

and we have the following result.

Theorem 2.2. If $\left(\mathrm{DLP}_{k+1}\right)$ is nondegenerate, then

$$
\begin{aligned}
V\left(\mathrm{LP}_{k+1}\right)-V\left(\mathrm{LP}_{k}\right) & =\sum_{j=1}^{n} \phi_{j}^{k+1} x_{j}^{k}-\sum_{i \in B_{k+1}^{k}} \phi_{k}\left(t_{i}\right) \mu_{k+1}\left(t_{i}\right) \\
& =\sum_{j=1}^{m_{k}} s_{j}^{k} \mu_{k}\left(A_{i_{j}^{k}}\right)-\sum_{j=1}^{n} \phi_{j}^{k} x_{j}^{k+1} .
\end{aligned}
$$

Proof. By the definition of $\phi_{k}(t)$, we have

$$
\begin{aligned}
& \sum_{i \in B_{k+1}^{\prime}} \phi_{k}\left(t_{i}\right) \mu_{k+1}\left(t_{i}\right) \\
= & \sum_{i \in B_{k+1}^{\prime}}\left(\sum_{j=1}^{n} f_{j}\left(t_{i}\right) x_{j}^{k}-g\left(t_{i}\right)\right) \mu_{k+1}\left(t_{i}\right) \\
= & \sum_{i \in B_{k+1}^{\prime}} \sum_{j=1}^{n} f_{j}\left(t_{i}\right) x_{j}^{k} \mu_{k+1}\left(t_{i}\right)-\sum_{i \in B_{k+1}^{\prime}} g\left(t_{i}\right) \mu_{k+1}\left(t_{i}\right) \\
= & \sum_{j=1}^{n}\left(\sum_{i \in B_{k+1}^{\prime}} f_{j}\left(t_{i}\right) \mu_{k+1}\left(t_{i}\right)\right) x_{j}^{k}-V\left(\mathrm{LP}_{k+1}\right) \\
= & \sum_{j=1}^{n}\left(\phi_{j}^{k+1}+c_{j}\right) x_{j}^{k}-V\left(\mathrm{LP}_{k+1}\right)
\end{aligned}
$$




$$
\begin{aligned}
& =\sum_{j=1}^{n} \phi_{j}^{k+1} x_{j}^{k}+\sum_{j=1}^{n} c_{j} x_{j}^{k}-V\left(\mathrm{LP}_{k+1}\right) \\
& =\sum_{j=1}^{n} \phi_{j}^{k+1} x_{j}^{k}+V\left(\mathrm{LP}_{k}\right)-V\left(\mathrm{LP}_{k+1}\right) .
\end{aligned}
$$

Therefore,

$$
V\left(\mathrm{LP}_{k+1}\right)-V\left(\mathrm{LP}_{k}\right)=\sum_{j=1}^{n} \phi_{j}^{k+1} x_{j}^{k}-\sum_{i \in B_{k+1}^{\prime}} \phi_{k}\left(t_{i}\right) \mu_{k+1}\left(t_{i}\right) .
$$

On the other hand, by the definition of $\phi_{j}^{k}$, we have

$$
\begin{aligned}
& \sum_{j=1}^{n} \phi_{j}^{k} x_{j}^{k+1} \\
& =\sum_{j=1}^{n}\left(\sum_{i \in B_{k}^{\prime}} f_{j}\left(t_{i}\right) \mu_{k}\left(t_{i}\right)-c_{j}\right) x_{j}^{k+1} \\
& =\sum_{j=1}^{n}\left(\sum_{i \in B_{k}^{\prime}} f_{j}\left(t_{i}\right) \mu_{k}\left(t_{i}\right) x_{j}^{k+1}\right)-\sum_{j=1}^{n} c_{j} x_{j}^{k+1} \\
& =\sum_{i \in B_{k}^{\prime}}\left(\sum_{j=1}^{n} f_{j}\left(t_{i}\right) x_{j}^{k+1}\right) \mu_{k}\left(t_{i}\right)-V\left(\mathrm{LP}_{k+1}\right) \\
& =\sum_{j=1}^{m_{k}}\left(s_{j}^{k}+g\left(t_{i}^{k}\right)\right) \mu_{k}\left(t_{i_{j}^{k}}\right)-V\left(\mathrm{LP}_{k+1}\right) . \\
& =\sum_{j=1}^{m_{k}} s_{j}^{k} \mu_{k}\left(t_{i_{j}^{k}}\right)+\sum_{t \in B_{k}^{\prime}} g\left(t_{i}\right) \mu_{k}\left(t_{i}\right)-V\left(\mathrm{LP}_{k+1}\right) \\
& =\sum_{j=1}^{m_{k}} s_{j}^{k} \mu_{k}\left(t_{i_{j}^{k}}\right)+V\left(\mathrm{LP}_{k}\right)-\mathrm{V}\left(\mathrm{LP}_{k+1}\right) .
\end{aligned}
$$

Hence, we have

$$
V\left(\mathrm{LP}_{k+1}\right)-V\left(\mathrm{LP}_{k}\right)=\sum_{j=1}^{m_{k}} s_{j}^{k} \mu_{k}\left(t_{i_{j}^{k}}\right)-\sum_{j=1}^{n} \phi_{j}^{k} x_{j}^{k+1} .
$$

Theorem 2.2 is a fundamental result; with it, we show that, under proper conditions, for any given $\delta>0$, the proposed scheme actually terminates in a finite number of iterations.

Theorem 2.3. Given any $\delta>0$, in each iteration, assume that :

(A1) $\left(\mathrm{LP}_{k}\right)$ has a bounded feasible domain;

(A2) $\left(\mathrm{DLP}_{k}\right)$ is nondegenerate. 
Moreover, there exists a $\bar{\delta}>0$ such that:
(A3) $\mu_{k}\left(t_{i}\right) \geq \bar{\delta}, \quad \forall i \in B_{k}^{\prime}$;
(A4) $\phi_{j}^{k}<-\bar{\delta}, \quad \forall j \notin B_{k}$;
(A5) $M_{k}^{T}$ has a square submatrix $D_{k}$ with rank $p_{k}\left(=\left|B_{k}\right|\right)$ and $\left|\operatorname{det}\left(D_{k}\right)\right|>\bar{\delta}$.

Then, the proposed scheme terminates in a finite number of iterations.

Proof. Suppose that the scheme does not stop in a finite number of iterations. When $\left(\mathrm{DLP}_{k}\right)$ is nondegenerate for each $k$, Theorem 2.1 implies that

$$
V\left(\mathrm{LP}_{1}\right)<V\left(\mathrm{LP}_{2}\right)<\cdots \leq V(\mathrm{LSIP})
$$

Thus,

$$
\lim _{r \rightarrow \infty} V\left(\mathrm{LP}_{r}\right)=\alpha \leq V(\mathrm{LSIP})
$$

We claim that this is impossible.

By (A1), the infinite sequence $\left\{x^{k}\right\}$ is confined in a compact set $C$ in $R^{n}$. There exists a subsequence $\left\{\mathrm{x}^{k}\right\}$ of $\left\{\mathrm{x}^{k}\right\}$ such that $\mathrm{x}^{k}$ converges to $\mathrm{x}^{*}$, and the subsequence $\left\{t_{k_{r}+1}\right\}$ converges to some point $t_{*}$ as $r \rightarrow \infty$. Now, we let

$$
\phi_{*}(t)=\sum_{i=1}^{n} f_{i}(t) x_{i}^{*}-g(t)
$$

Then, $\phi_{k_{r}}\left(t_{k_{r}+1}\right)$ converges to $\phi_{*}\left(t_{*}\right)$. Since $\phi_{k_{r}}\left(t_{k_{r}+1}\right)<-\delta$, for each $r$, we have $0 \neq \phi_{*}\left(t_{*}\right) \leq-\delta$.

Now, let $\epsilon \in(0, \delta)$ be an arbitrary number; we can find a large integer $N \in\left\{k_{r}\right\}_{r=1}^{\infty}$ such that

$$
\left|V\left(\mathrm{LP}_{N}\right)-\alpha\right| \leq \epsilon^{2}, \quad\left|\phi_{N}\left(t_{N+1}\right)-\phi_{*}\left(t_{*}\right)\right| \leq \epsilon^{2} .
$$

By Theorem 2.2, we have

$$
\left|V\left(\mathrm{LP}_{N+1}\right)-V\left(\mathrm{LP}_{N}\right)\right|=\left|\sum_{j=1}^{m_{N}} s_{j}^{N} \mu_{N}\left(t_{i},{ }^{N}\right)-\sum_{j=1}^{n} \phi_{j}^{N} x_{j}^{N+1}\right| \leq \epsilon^{2}
$$

Recall that

$$
\phi_{j}^{N} \leq 0, \quad j=1,2, \ldots, n
$$


each term in the first summation sign of (33) is nonnegative, and each term in the second summation sign of (33) is nonpositive. It follows that

$$
\begin{array}{ll}
0 \leq s_{j}^{N} \mu_{N}\left(t_{i j}^{N}\right) \leq \epsilon^{2}, & j=1, \ldots, m_{N}, \\
0 \leq-\phi_{j}^{N} x_{j}^{N+1} \leq \epsilon^{2}, & j=1, \ldots, n .
\end{array}
$$

By (A3), we have

$$
\mu_{N}\left(t_{i}\right) \leq \bar{\delta}, \quad \forall i \in B_{N}^{\prime} .
$$

Hence,

$$
s_{j}^{N} \leq \epsilon, \quad j=1, \ldots, m_{N} .
$$

By (A4), we have

$$
\phi_{j}^{N}<-\bar{\delta}, \quad j \notin B_{N} .
$$

It follows from (33) that

$$
x_{j}^{N+1} \leq \epsilon, \quad j \notin B_{N} .
$$

By (29), (34), and (36), we have

$$
\sum_{j \in B_{N}} f_{j}\left(t_{i}\right) x_{j}^{N+1}=g\left(t_{i}\right)+O_{i}(\epsilon), \quad i \in B_{N}^{\prime},
$$

where $O_{i}(\epsilon) \rightarrow 0$ as $\epsilon \rightarrow 0$.

By (24), we have

$$
\sum_{j \in B_{N}} f_{j}\left(t_{i}\right) x_{j}^{N}=g\left(t_{i}\right), \quad i \in B_{N}^{\prime} .
$$

By the definition of $M_{N}$, we know that the matrix $M_{N}$ has row vectors

$$
\left(f_{j}\left(t_{i_{1}^{N}}\right), f_{j}\left(t_{i_{2}^{N}}\right), \ldots, f_{j}\left(t_{i_{m_{N}}^{N}}\right)\right), \quad j \in B_{N} .
$$

Let

$$
\underline{\mathrm{x}}=\left(x_{j}^{N}\right)_{j \in B_{N}}, \quad \underline{\mathrm{x}}^{\prime}=\left(x_{j}^{N+1}\right)_{j \in B_{N}} .
$$

Then, (37) and (38) can be expressed as

$$
\begin{aligned}
& M_{N \underline{\mathrm{x}}^{\prime}}^{T}=\left(g\left(t_{i_{1}^{N}}^{N}\right)+O_{1}(\epsilon), \ldots, g\left(t_{i_{m_{N}}^{N}}\right)+O_{m_{N}}(\epsilon)\right)^{T}, \\
& M_{N \underline{\mathrm{x}}}^{T}=\left(g\left(t_{i_{1}^{N}}\right), \ldots, g\left(t_{i_{N}^{*}}\right)\right)^{T} .
\end{aligned}
$$

It follows from (A5) that

$$
\left|x_{j}^{N+1}-x_{j}^{N}\right| \leq \epsilon^{*}(\epsilon), \quad j \in B_{N},
$$

where $\epsilon^{*}(\epsilon) \rightarrow 0$ as $\epsilon \rightarrow 0$. 
Combining the facts that $\phi_{N+1}\left(t_{N+1}\right)=0,(36)$, and (39), we have

$$
\phi_{N}\left(t_{N+1}\right) \rightarrow 0, \quad \text { as } \epsilon \rightarrow 0 .
$$

But

$$
\phi_{N}\left(t_{N+1}\right) \rightarrow \phi_{*}\left(t_{*}\right) \neq 0, \quad \text { as } N \rightarrow \infty .
$$

Hence, $(40)$ cannot be true and we have a contradiction. Therefore, our claim is valid and the proof is complete.

Note that (Al) is commonly assumed in linear semi-infinite programming to simplify proofs. It can be relaxed by using bounded level sets. (A2) is also a technical condition commonly used in linear programming. Moreover, when $\delta$ is chosen to be sufficiently small, (A3), (A4), (A5) in general can be satisfied without much difficulty. The violation of any of these three assumptions will lead to some rare instances of degeneracy or inconsistency. In particular, the violation of (A3) will result in a subsequence of $\left(D P_{k}\right)$ which has a limit optimal solution being dual degenerate. A similar situation goes for the violation of (A4). Moreover, the violation of (A5) will provide a subsequence of $\left(\mathrm{LP}_{k}\right)$ whose determinant value of optimal basis matrix tends to zero.

Under these conditions, Theorem 2.3 assures that the proposed scheme terminates in finitely many iterations, say $k^{*}$ iterations, with an optimal solution

$$
\mathrm{x}^{k^{*}}=\left(x_{1}^{k^{*}}, \ldots, x_{n}^{k^{*}}\right)^{T}
$$

such that

$$
x_{j}^{k^{*}}>0, \quad j \in B_{k^{*}} .
$$

In this case, $x^{k^{*}}$ is of course feasible for (LSIP), and it can be viewed as an approximate solution of (LSIP). The next theorem tells us how good such approximate solution can be.

Theorem 2.4. For any given $\delta>0$, if there exists $\overline{\mathrm{x}}=\left(\bar{x}_{1}, \ldots, \bar{x}_{n}\right)^{r}$, with $\bar{x}_{j} \geq-x_{j}^{k^{*}} / \delta, \forall j \in B_{k^{*}}$, and $\bar{x}_{j} \geq 0, \forall j \notin B_{k^{*}}$, such that

$$
\sum_{j=1}^{k} \bar{x}_{j} f_{j}(t) \geq 1, \quad \forall t \in T
$$

then

$$
\left|V\left(\mathrm{LP}_{k^{*}}\right)-V(\operatorname{LSIP})\right| \leq \delta\left|\sum_{j=1}^{n} c_{j} \bar{x}_{j}\right|
$$


Proof. By the definition of $\mathrm{x}^{k^{*}}$, we have

$$
\sum_{j=1}^{n} f_{j}(t) x_{j}^{k^{*}}-g(t) \geq-\delta, \quad \forall t \in T
$$

By (41), we have

$$
\sum_{j=1}^{n} f_{j}(t) \delta \bar{x}_{j} \geq \delta, \quad \forall t \in T
$$

It follows from (42) and (43) that

$$
\sum_{j=1}^{n} f_{j}(t)\left(x_{j}^{k^{*}}+\delta \bar{x}_{j}\right)-g(t) \geq 0, \quad \forall t \in T
$$

By our assumption, we know that

$$
x_{j}^{k^{*}}+\delta \bar{x}_{j} \geq 0, \quad j=1,2, \ldots, n .
$$

Hence, $\mathrm{x}^{k^{*}}+\delta \overline{\mathrm{x}}$ is a feasible solution of (LSIP). Therefore,

$$
\begin{aligned}
\left|V\left(\mathrm{LP}_{k^{*}}\right)-V(\operatorname{LSIP})\right| & \leq\left|\sum_{j=1}^{n} c_{j} x_{j}^{k^{*}}-\sum_{j=1}^{n} c_{j}\left(x_{j}^{k^{*}}+\delta \bar{x}_{j}\right)\right| \\
& =\delta\left|\sum_{j=1}^{n} c_{j} \bar{x}_{j}\right| .
\end{aligned}
$$

Note that the required condition (41) implies that (1) has an interior solution. Also, when $\delta>0$ is chosen to be small enough, since $\left\{f_{j}(t) \mid j \in B_{k^{*}}\right\}$ are in general linearly independent, the existence of the required $\overline{\mathbf{x}}$ in Theorem 2.4 is not a problem. Theorems 2.3 and 2.4 guarantee that the proposed scheme generates an approximate solution to (LSIP) with any level of accuracy in a finite number of iterations.

\section{Computational Experiments}

In this section, the following two commonly seen $\mathrm{Ll}$ problems (Refs. 3 and 16) are used to illustrate the computational behavior of the proposed scheme: 
Problem 3.1.

$$
\begin{array}{ll}
\max & \sum_{j=1}^{8}\left(-w_{j} / j\right) \\
\text { s.t. } & \sum_{j=1}^{8}\left(-t^{j-1} w_{j}\right) \leq-1 /(2-t), \quad t \in[0,1] .
\end{array}
$$

Problem 3.2.

$$
\begin{array}{ll}
\max & \sum_{j=1}^{7}\left(-w_{j} / j\right) \\
\text { s.t. } & \sum_{j=1}^{7}\left(-t^{j-1} w_{j}\right) \leq \sum_{j=0}^{4} t^{2 j}, \quad t \in[0,1] .
\end{array}
$$

It was reported in Refs. 3 and 16 that 0.6931 and -1.78688 are approximate optimal solutions to Problems 3.1 and 3.2, respectively.

We use Matlab Version 4.2c (Ref. 17) on a SUN UltraSparcl workstation for the experiment. Three different approaches (namely, the tradjtional cutting plane method, the proposed method, and the well-known discretization method) have been implemented.

For all three methods, the lp subroutine of the MATLAB optimization toolbox was used to solve linear programs in each of them. For the discretization method, we discretized the interval $[0,1]$ into 101 evenly spaced points and solved a linear program with 101 explicit constraints. For the traditional cutting plane method, the $f_{\min }$ subroutine of MATLAB was utilized for finding the global minimizer of problem (8).

For the proposed method, we set $\delta=0.0001$ and recursively discretize $T$ to find $t_{k+1}$ in Step 3. In other words, in the beginning, the interval $[0,1]$ is discretized by 11 evenly spaced points, and we test each point to see if $\phi(t)<-\delta$. If all points failed, then we refine the discretization by 101 points to test. If this failed, then we test 1001 points. If this failed again, the MATLAB subroutine $f_{\min }$ is finally employed to find $t_{k+1}$. Our numerical experiment has shown that only in the last iteration is the subroutine $f_{\min }$ called for the proposed method.

In our experiment, the condition

$$
\phi_{k}\left(t_{k+1}\right)>-0.0001
$$

was used as a stopping criterion for both the traditional and proposed methods. Also notice that the relaxed scheme was stated in Section 2 as a theoretical algorithm, without loss of generality, under the assumption that 
Table 1. Comparison of different methods.

\begin{tabular}{|c|c|c|c|c|c|c|}
\hline & \multicolumn{3}{|c|}{ Problem 3.1} & \multicolumn{3}{|c|}{ Problem 3.2} \\
\hline & Obj. value & $k$ & $\begin{array}{l}\text { Time } \\
(\mathrm{sec})\end{array}$ & Obj. value & $k$ & $\begin{array}{l}\text { Time } \\
(\mathrm{sec})\end{array}$ \\
\hline Traditional cp method & 0.693146 & 14 & 6.87 & $-2.2395 \mathrm{e}+11$ * & 71 & 40.96 \\
\hline Proposed cp method & 0.693148 & 12 & 4.82 & -1.786917 & 14 & 4.94 \\
\hline Discretized method & 0.693148 & & 6.18 & -1.786901 & & 1.46 \\
\hline
\end{tabular}

* The algorithm does not converge in 70 iterations.

$\left(\mathrm{LP}_{k}\right)$ is solvable with an optimal solution $\mathrm{x}^{k}$. In practice, even when (LSIP) is solvable, some $\left(\mathrm{LP}_{k}\right)$ at the early stages may still be unbounded below. This problem can be handled easily by adding enough points to $T_{1}$ to start the first iteration; or we can simply choose $x^{k}$ in Step 2 to be a feasible solution of $\left(\mathrm{LP}_{k}\right)$, whose objective value is negatively large. In our experiments, we adopted the latter approach. The numerical results are shown in Table 1.

In the table, obj. value is the final objective value, $k$ indicates the number of iterations required, and time is in CPU-seconds. Note that $k$ also represents the number of linear programs (or number of explicit constraints) solved. For both problems, the proposed method generates a better approximate solution (comparable to the result obtained by the discretization method with 100 constraints) in shorter time than the traditional cutting plane method. For Problem 3.2, the traditional cutting plane method failed to find an approximate solution in 70 iterations. In this case, all optimizers (i.e., $t_{k}^{\prime} s$ ) generated by the traditional cutting plane method stay quite close, so the traditional method failed to converge fast. This experiment shows the potential of the proposed method.

To further understand the role that $\delta$ played in the proposed method, we ran the proposed method for both problems with different $\delta$ values. The results are shown in Table 2.

In theory for the proposed method, we think that $\delta$ should be very small, such as $10^{-7}$ for our workstation implementation. But in practice, $\delta$

Table 2. Numerical behaviors of using different $\delta$.

\begin{tabular}{lcccccccc}
\hline & \multicolumn{3}{c}{ Problem 3.1 } & & \multicolumn{3}{c}{ Problem 3.2 } \\
\cline { 2 - 4 } \cline { 7 - 9 } \multicolumn{1}{c}{} & Obj. value & $k$ & Time $(\mathrm{sec})$ & & Obj. value & $k$ & Time (sec) \\
\hline 0.0001 & 0.693148 & 12 & 4.82 & & -1.786917 & 14 & 4.94 \\
0.01 & 0.693148 & 12 & 4.75 & & -1.787018 & 10 & 2.95 \\
1.0 & 0.693148 & 11 & 4.46 & & -1.787018 & 9 & 2.75 \\
\hline
\end{tabular}


can be chosen much larger than we expected. In our experiment, the results are not bad even when $\delta=1.0$.

\section{Explicit Algorithm}

The proposed scheme adds one inequality constraint in each iteration. Hence, the size of $\left(\mathrm{LP}_{k}\right)$ becomes larger and larger. In order to avoid solving too large problems, we would like to exploit the possibility of dropping unnecessary constraints, while a new constraint is added in each iteration. This is referred to as an explicit exchange method in this paper.

To introduce such an explicit algorithm, let us start with some notation. Let $T^{\prime}=\left\{t_{1}, \ldots, t_{m}\right\}$ be a subset with $m$ elements in $T$. We denote by (LP $\left.\left(T^{\prime}\right)\right)$ the following linear program with $m$ explicit constraints induced by $T^{\prime}$ :

$$
\begin{aligned}
\left(\operatorname{LP}\left(T^{\prime}\right)\right) \quad \min & \sum_{j=1}^{n} c_{j} x_{j}, \\
\text { s.t. } & \sum_{j=1}^{n} x_{j} f_{j}\left(t_{i}\right) \geq g\left(t_{i}\right), \quad i=1,2, \ldots, m, \\
& x_{j} \geq 0, \quad j=1,2, \ldots, n .
\end{aligned}
$$

Similar to the situation faced in Section 2, for the purpose of easy description, given that $\delta>0$ is a prescribed small number, we state our explicit algorithm in the following steps, under the assumption that $\operatorname{LP}\left(T_{k}\right)$ and its dual $\operatorname{DLP}\left(T_{k}\right)$ are both solvable.

Step 0 . Let $k \leftarrow 1$, choose any $t_{1}^{0} \in T$, set $T_{1}=\left\{t_{1}^{0}\right\}$, and set $m_{0}=0$.

Step 1. Solve $\operatorname{LP}\left(T_{k}\right)$. Let $\mathrm{x}^{k}=\left(x_{1}^{k}, \ldots, x_{n}^{k}\right)^{T}$ be an optimal solution. Define $\phi_{k}(x)$ according to Eq. (7).

Step 2. Solve $\operatorname{DLP}\left(T_{k}\right)$. Let $\mathrm{y}^{k}=\left(y_{1}^{k}, \ldots, y_{m_{k}+1}^{k}\right)^{T}$ be an optimal solution. Define a discrete measure $\mu_{k}$ on $T$ by letting

$\mu_{k}(t)= \begin{cases}y_{i}^{k}(\geq 0), & \text { if } t=t_{i}^{k-1} \in T_{k}, \\ 0, & \text { if } t \in T \backslash T_{k} .\end{cases}$

Set $E_{k}=\left\{t \in T_{k} \mid \mu_{k}(t)>0\right\}=\left\{t_{1}^{k}, \ldots, t_{m_{k}}^{k}\right\}$.

Step 3. Find any $t_{m_{k}+1}^{k} \in T$ such that $\phi_{k}\left(t_{m_{k}+1}^{k}\right)<-\delta$.

If such $t_{m_{k}+1}^{k}$ does not exist, stop and output $\mathrm{x}^{k}$ as a solution. Otherwise, set $T_{k+1}=E_{k} \cup\left\{t_{m_{k}+1}^{k}\right\}$.

Step 4. Update $k \leftarrow k+1$, and go to Step 1 . 
Note that $t_{m_{k}+1}^{k} \notin T_{k}$ and, if the algorithm terminates in Step 3, then the output solution $\mathrm{x}^{k}$ indeed solves (LSIP), with $\delta$ being sufficiently small. Also note that, when it is applicable, a primal-dual algorithm can be employed to find solutions for $\operatorname{LP}\left(T_{k}\right)$ and $\operatorname{DLP}\left(T_{k}\right)$ simultaneously for computational efficiency.

Recall that

$$
\mathrm{x}^{k}=\left(x_{1}^{k}, \ldots, x_{n}^{k}\right)^{T}
$$

solves $\operatorname{LP}\left(T_{k}\right)$. Let

$$
\boldsymbol{B}_{k}=\left\{j_{1}^{k}, \ldots, j_{p_{k}}^{k}\right\}
$$

be an index set such that $x_{j}^{k}>0$, if and only if $j \in B_{k}$. Also, let $M_{k}$ be a $p_{k} \times m_{k}$ matrix with its $j$ th row vector being

$$
\left(f_{j}\left(t_{1}^{k}\right), \ldots, f_{j}\left(t_{m_{k}}^{k}\right)\right), \quad j \in B_{k} .
$$

On the dual side, recalling the definition of $\phi_{k}(t)$, we define

$$
\phi_{j}^{k}=\sum_{i=1}^{m_{k}} f_{j}\left(t_{i}^{k}\right) \mu_{k}\left(t_{i}^{k}\right)-c_{j}, \quad j=1, \ldots, n .
$$

In this way, we know that

$$
\phi_{j}^{k} \leq 0, \quad \forall j, k \text {. }
$$

With this setting, and parallel to the proof given in Theorem 2.3, we have the following result.

Theorem 4.1. Given any $\delta>0$, in each iteration, assume that:

(A1) The set $\left\{\mathrm{x}^{1}, \mathrm{x}^{2}, \ldots\right\}$ is bounded;

(A2) $\operatorname{DLP}\left(T_{k}\right)$ is nondegenerate.

Moreover, there exists a $\bar{\delta}>0$ such that:

(A3) $\mu_{k}\left(t_{i}^{k}\right) \geq \bar{\delta}, \forall i=1, \ldots, m_{k}$;

(A4) $\phi_{j}^{k}<-\bar{\delta}, \forall j \notin B_{k}$;

(A5) $M_{k}^{T}$ has a square submatrix $D_{k}$ with rank $p_{k}\left(=\left|B_{k}\right|\right)$ and $\left|\operatorname{det}\left(D_{k}\right)\right|>\bar{\delta}$.

Then, the explicit algorithm terminates in a finite number of iterations.

The above theorem asserts that, under proper assumptions, the explicit algorithm terminates with a solution in a finite number of iterations. Similarly, the result of Theorem 2.4 can be used to tell how good such a solution is. 
JOTA: VOL. 99, NO. 3, DECEMBER 1998

Table 3. Comparison of relaxed scheme and explicit algorithm, Problem 3.2.

\begin{tabular}{lcc}
\hline & \multicolumn{2}{c}{ Number of constraints in $\left(\mathrm{LP}\left(T_{k}\right)\right)$} \\
\cline { 2 - 3 } Iteration $k$ & Relaxed scheme & Explicit algorithm \\
\hline 19 & 19 & $1-9$ \\
10 & 10 & 6 \\
11 & 11 & 6 \\
12 & 12 & 6 \\
13 & 13 & 6 \\
14 & 14 & 6 \\
\hline Time (sec) & 4.94 & 4.73 \\
Obj. value & -1.786917 & -1.786917 \\
\hline
\end{tabular}

Note that, for the relaxed scheme in Theorem 2.3 , if $\left(\mathrm{LP}_{k}\right)$ has a bounded feasible domain, so do $\left(\mathrm{LP}_{k+1}\right),\left(\mathrm{LP}_{k+2}\right), \ldots$ However, for the explicit algorithm, $T_{k}$ may not be a subset of $T_{k+1}$. Therefore, we assume directly that the set of optimal solutions to $\operatorname{LP}\left(T_{k}\right), k=1,2, \ldots$, is bounded.

We now use Problem 3.2 to illustrate the potential of the explicit algorithm. The computational experiment was conducted in the same test environment as described in Section 3, and the result is shown in Table 3. Observe that, in the first nine iterations, since $\operatorname{LP}\left(T_{k}\right)$ is unbounded below and hence $\operatorname{DLP}\left(T_{k}\right)$ is infeasible, we simply find one feasible solution of $\operatorname{LP}\left(T_{k}\right)$ and define $\phi_{k}(x)$ in Step 1, set $E_{k}=T_{k}$, and jump to Step 3. In this way, both methods add one constraint in each iteration. After the 9th iteration, $\operatorname{DLP}\left(T_{k}\right)$ becomes feasible and the explicit algorithm starts dropping unnecessary constraints. In this experiment, both cases stop at the 14th iteration with literally the same final objective value. Although the time reduction is not huge for this small problem, the potential of the explicit algorithm is clearly seen.

\section{Concluding Remarks}

In this paper, we have presented a relaxed cutting plane scheme to solve linear semi-infinite programming problems. In each iteration, the proposed scheme chooses a point where the infinite constraints are violated to a degree rather than a point where the violations are maximized to generate a new cut for the next iteration. Under proper conditions, it has been proven that the proposed scheme can generate an approximate solution of any level of accuracy in a finite number of iterations. Based on this scheme, an explicit 
algorithm which allows the dropping of unnecessary constraints is developed.

Since the requirement of finding an optimizer of a nonlinear and nonconvex program is relaxed, we see the potential advantage of the proposed scheme, in particular, in higher-dimensional spaces. Our very limited computational results support the theory. Although the method that we implemented for finding $t_{k+1}$ in Step 3 of the proposed scheme is very primitive, and may not be good for general purposes, the potential advantage of the proposed method is clearly illustrated.

\section{References}

1. Anderson, E. J., and NAsh, P., Linear Programming in Infinite-Dimensional Spaces, Wiley, Chichester, England, 1987.

2. LaI, H. C., and Wu, S. Y., On Linear Semi-Infinite Programming Problems: An Algorithm, Numerical Functional Analysis and Optimization, Vol. 13, pp. 287304, 1992.

3. Glashoff, K., and Gustafson, S. A., Linear Optimization and Approximation, Springer Verlag, New York, New York, 1982.

4. Gustafson, S. A., and Kortanek, K. O., Semi-Infinite Programming and Applications, Mathematical Programming: The State and Art, Edited by A. Bachem, M. Grotschel, and B. Korte, Springer Verlag, Berlin, Germany, pp. 132-157, 1983.

5. Gustafson, S. $\AA$., On the Computational Solution of a Class of Generalized Moment Problems, SIAM Journal on Numerical Analysis, Vol. 7, pp. 343-357, 1970.

6. Gustafson, S. $\AA$., and Kortanek, K. O., Numerical Treatment of a Class of Semi-Infinite Programming Problems, Naval Research Logistics Quarterly, Vol. 20, pp. 473-504, 1973.

7. Hettich, R., and Kortanek, K. O., Semi-Infinite Programming: Theory, Method, and Applications, SIAM Review, Vol. 35, pp. 380-429, 1993.

8. FANG, S. C., and WU, S. Y., An Inexact Approach to Solving Linear Semi-Infinite Programming Problems, Optimization, Vol. 28, pp. 291-299, 1994.

9. Fang, S. C., Lin, C. J., and Wu, S. Y., On Solving Convex Quadratic SemiInfinite Programming Problems, Optimization, Vol. 31, pp. 107-125, 1994.

10. Gribik, P. R., A Central Cutting Plane Algorithm for SIP, Semi-Infinite Programming. Edited by R. Hettich, Springer Verlag, Berlin, Germany, pp. 66-82, 1979.

11. Tichatschke, R., and Nebeling, V., A Cutting Plane Algorithm for Solving Quadratic Semi-Infinite Programs, Optimization, Vol. 19, pp. 803-817, 1988.

12. Gustafson, S. A., and Kortanek, K. O., Computation of Optimal Experimental Designs for Air Quality Surveillance, Quantitative Modelle für Ökonomisch-Ökologische Analysen, Edited by P. J. Jansen, O. Moeschlin, and O. Rentz, Verlag Anton Hain, Meisenheim, Germany, pp. 43-60, 1976. 
13. Gustafson, S. A., and Kortanek, K. O., Computational Schemes for SemiInfinite Programs, Technical Report, Department of Mathematics, CarnegieMellon University, Pittsburgh, Pennsylvania, 1977.

14. Fang, S. C., and Puthenpura, S. C., Linear Optimization and Extensions: Theory and Algorithms, Prentice Hall, Englewood Cliffs, New Jersey, 1993.

15. Luenberger, D. G., Linear and Nonlinear Programming, Addison-Wesley, Reading, Massachusetts, 1984.

16. Ferris, M. C., and Philpott, A. B., An Interior-Point Algorithm for SemiInfinite Linear Programming, Mathematical Programming, Vol. 43, pp. 257-276, 1989.

17. Matlab User Guide, The Math Works, Natick, Massachusetts, 1994. 